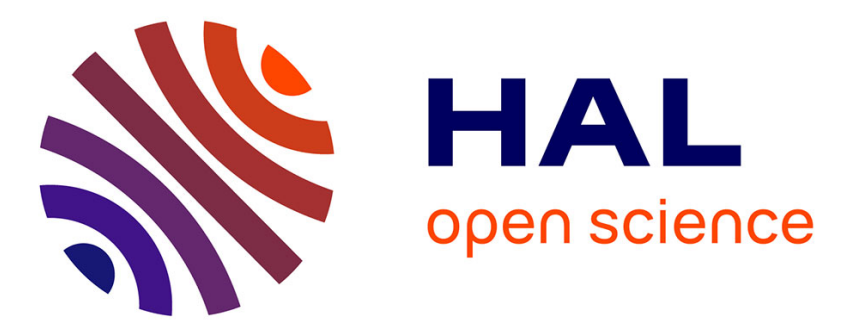

\title{
Guru et politique en Inde. Des éminences grises à visage découvert?
}

Christophe Jaffrelot

\section{To cite this version:}

Christophe Jaffrelot. Guru et politique en Inde. Des éminences grises à visage découvert?. Politix, 2001, 14 (54), pp.75-94. 10.3406/polix.2001.1155 . hal-01016935

\section{HAL Id: hal-01016935 \\ https://hal-sciencespo.archives-ouvertes.fr/hal-01016935}

Submitted on 1 Jul 2014

HAL is a multi-disciplinary open access archive for the deposit and dissemination of scientific research documents, whether they are published or not. The documents may come from teaching and research institutions in France or abroad, or from public or private research centers.
L'archive ouverte pluridisciplinaire HAL, est destinée au dépôt et à la diffusion de documents scientifiques de niveau recherche, publiés ou non, émanant des établissements d'enseignement et de recherche français ou étrangers, des laboratoires publics ou privés. 


\section{Guru et politique en Inde. Des éminences grises à visage} découvert?

In: Politix. Vol. 14, N54. Deuxième trimestre 2001. pp. 75-94.

Citer ce document / Cite this document :

Jaffrelot Christophe. Guru et politique en Inde. Des éminences grises à visage découvert ?. In: Politix. Vol. 14, N54. Deuxième trimestre 2001. pp. 75-94.

doi : 10.3406/polix.2001.1155

http://www.persee.fr/web/revues/home/prescript/article/polix_0295-2319_2001_num_14_54_1155 


\section{Résumé}

Guru et politique en Inde : des éminences grises à visage découvert?

Christophe Jaffrelot

La configuration classique du pouvoir en Inde associe au souverain un conseiller spirituel de caste brahmane. Ce schéma traditionnel a perdu tout droit de cité dans le cadre de la sécularisation de l'Etat après 1947. Néanmoins, des hommes politiques, comme Indira Gandhi, ont continué d'entretenir d'étroites relations avec leur guru et ont volontiers rendues publiques ces relations lorsque cela améliorait leur popularité. En fait, de telles relations se révélaient illégitimes lorsque les guru en question étaient des tantriques ou lorsqu'ils entretenaient des liens illicites avec le monde criminel. Le rapport au pouvoir qu'entretient le RSS (Rashtriya swayamsevak sangh, Association des volontaires nationaux) est calqué sur le modèle traditionnel puisque cette organisation se veut le guru du pouvoir. L'accès de " son » parti politique, le BJP, au gouvernement lui en donne la possibilité depuis 1998. Après s'être contenté de conseiller le pouvoir en coulisses, le RSS sort de plus en plus de l'ombre en partie parce que le premier ministre Vajpayee peut trouver avantage à se prévaloir de ses liens privilégiés avec une organisation connue pour sa pureté doctrinale et son intégrité. Le caractère secret des guru modernes est donc à géométrie variable, que les guru en question soient des individus ou, comme dans le cas du RSS, des collectifs.

\section{Abstract}

Guru and Politics in India: Uncovered "Eminences Grises" ?

Christophe Jaffrelot

In ancient India, the Hindu king was guided by a spiritual counsellor, a guru. This tradition receded in the background after independence because of the secularisation of the state. Yet, politicians, like Indira Gandhi, have continued to cultivate close relationships with guru, and have even occasionnally publicised this relationship when such a move helped them to improve their image. Such relationships were not legitimate in two cases only, that of the tantric guru and that of men with some underground connection. The conception of power that the RSS (Rashtriya Swayamsevak Sangh, Association of National Volunteers) has developed directly derives from the traditional pattern of a king and his guru. Since its inception, the RSS aimed at becoming the Raj guru of the Indian state. The rise to power of the BJP in 1998 enables it to play such a role today. After doing so in the background for long, the RSS is now coming forward because the Prime minister Atal Bihari Vajpayee try to benefit from his association with an organisation which is well known for its Hindu nationalist ideology and integrity. The secrecy of the relation between politicians and guru therefore, is not imperative. It may be publicised for strengthening the legitimacy of the former. 


\section{Guru et politique en Inde}

Des éminences grises à visage découvert?

Christophe JAFFRELOT

i7) ans une de ses tentatives pour caractériser le politique en Inde, Ashis Nandy soutient que : "La spécificité de la notion indienne du pouvoir réside dans ses connotations fortement "privées". Les Cormes les plus respectées du pouvoir sont le pouvoir sur soi - la maîtrise de soi, le contrôle de ses instincts et de ses appétits matériels. " Il ajoute dans le même esprit: "Les gouvernants sont sans cesse invités à se prêter au discours de l'ascétisme et du sacrifice, pour faire même de la plus triviale de leurs activités politiques l'élément d'un grand dessein moral - comme si le pouvoir sur soi et sur ses instincts légitimait le pouvoir politique ${ }^{1}$. " Nandy fait indirectement référence ici à la notion de swaraj, qui désigne en Inde la maîtrise spirituelle d'un individu sur lui-même mais aussi, dans le vocabulaire politique du mouvement de libération anti-colonial, le contrôle de son destin national, c'est-à-dire l'indépendance. Ce principe est au cœur de la démarche gandhienne puisque le Mahatma (la "grande âme ") cherchait à la fois la maîtrise spirituelle et l'émancipation de la tutelle britannique - d'où l'ambivalence de ce renonçant en politique, partagé entre

1. Nandy (A.), At the Edge of Psychology. Essay in Politics and Culture, Delhi, Oxford University Press, 1980, p. 50 et 51 . 
une quête personnelle et une ambition publique, comme si la maîtrise de soi était la condition d'une mobilisation politique réussie ${ }^{2}$.

Est-ce vraiment là la configuration typique du politique en Inde, comme le suggère Nandy? Faut-il considérer cette dualité entre le privé et le public sous l'aspect d'un masque de Janus? En fait, les deux faces en question ne sont pas nécessairement réunies dans un même individu, comme cela a été le cas pour Gandhi. Les hommes politiques indiens, loin de poursuivre une quête spirituelle par eux-mêmes, s'adjoignent volontiers les services d'un guru, se conformant en cela aux canons de la tradition hindoue. Celle-ci prévoyait en effet que le souverain fût flanqué d'un chapelain dont la magistrature morale lui assurait un droit de regard sur les affaires de l'Etat. Comment cette configuration a-t-elle survécu à la sécularisation de la sphère publique dont témoigne l'introduction de la laïcité dans la Constitution de 1950 ? Les politiques doivent-ils désormais cacher leurs guru ou peuvent-ils encore les montrer? Il semble en fait qu'il leur faille parfois les dissimuler et parfois veiller à ce que leur existence soit connue du grand public. La part de l'ombre est tout simplement à géométrie variable parce que l'association avec un guru peut renforcer la légitimité des politiques qui savent en user habilement. La montée en puissance du mouvement nationaliste hindou a confirmé cette éventualité, même si, en l'occurrence, le guru en question est d'un genre nouveau.

\section{Les conseillers du prince : des figures secrètes qu'il faut savoir rendre publiques}

\section{Le modèle traditionnel}

Dans le modèle classique de l'Etat indien, le souverain, de caste kshatriya (la caste de guerriers), doit être associé à un brahmane (un membre de la caste sacerdotale) qui cautionne son statut et lui sert de conseiller - voire qui le légitime parce qu'il inspire son action. En effet, le roi est guidé par le purohita ou «celui qui vient devant» pour les affaires qui regardent le dharma (l'ordre socio-cosmique, c'est-à-dire celui du monde des castes et celui du cosmos qui entretient avec le premier un rapport homothétique). Selon Robert Lingat : «Le dharma ne peut être réalisé que par leur coopération : le brahmane est le véritable cerveau du roi » parce que, dans les Dharmashastra (les traités du Dharma, textes normatifs de la littérature brahmanique), « le roi, en raison même de la puissance dont il est investi et qui lui permet de tout faire, ne doit prendre de décision qu'après avoir requis les conseils de

2. Je me permets de renvoyer le lecteur intéressé par cette question à la deuxième partie de Jaffrelot (C.), La démocratie en Inde. Religion, caste et politique, Paris, Fayard, 1998 ("Le Saint et le politique "). 
personnes compétentes et éclairées, au premier rang desquelles sont naturellement les brahmanes ${ }^{3}$ ". D'après les textes de 1'Inde ancienne, le souverain n'exerçait donc pas de droit l'autorité suprême: il lui fallait la caution des brahmanes qui incarnaient les valeurs les plus hautes et qui attestaient l'essence royale des kshatriya ou, au contraire, la leur déniait au moyen de généalogies de leur $\mathrm{cru}^{4}$.

Dans la pratique des royaumes hindous jusqu'à l'époque coloniale, les choses étaient quelque peu différentes. Si la logique théorique du système des castes reconnaissait aux brahmanes un statut supérieur aux kshatriya, le souverain s'émancipait facilement de la tutelle de son " chapelain 5 » dans tous les domaines dénués de connotations religieuses. En outre, le brahmane vivait des largesses de son " patron " : il était du devoir du roi de se montrer généreux à son égard et de protéger sa personne ainsi que ses biens terrestres. Le brahmane se trouvait ainsi dans une position de dépendance matérielle vis-à-vis du souverain. Nicholas Dirks écrit à ce propos que "jusqu'à la colonisation britannique, dans l'Inde du sud, la couronne [des rois] n'était pas aussi creuse qu'on ne l'a généralement laissé entendre. Les rois n'étaient pas inférieurs aux brahmanes ${ }^{6}$ ». La subordination du roi au brahmane était donc relative et variait selon les situations. Mais la pratique recoupe la théorie d'un point de vue essentiel puisqu'elle confirme la différenciation des sphères de l'autorité spirituelle et du pouvoir temporel. Ces deux domaines s'incarnent dans des personnages emblématiques et complémentaires du point de vue de la tradition hindoue, comme leur nom l'indique d'ailleurs, puisqu'on parle de Rajah (roi) et de Raj guru (guru du roi), le premier étant un kshatriya et le second un brahmane.

Cette configuration du pouvoir ne se rencontre pas qu'en Inde, comme A. M. Hocart l'a bien montré en comparant l'Inde et différents royaumes

3. Lingat (R.), Les sources du droit dans le système traditionnel de l'Inde, Paris, Mouton, 1967, p. 240242. Sur le même sujet, cf. aussi Coomaraswamy (A.), Autorité spirituelle et pouvoir temporel dans la perspective indienne du gouvernement, Milan, Arché, 1985. Selon A. M. Hocart, le conseiller spirituel du roi, en étant à la fois son « directeur de conscience " et son « spécialiste en matière de rituel ", détient les clefs de la prospérité du pays et « devient nécessairement l'homme le plus influent du royaume. En définitive, le roi dirige la politique, ce qui consiste en grande partie à appliquer les règles établies par les prêtres pour obtenir la prospérité " (Hocart (A. M.), Rois et courtisans, Paris, Le Seuil, 1978, p. 259).

4. Cf. Reiniche (M.-L.), «A propos d'une zamindari du Tamilnad. En quel sens peut-on parler d'Etat? ", in Reiniche (M.-L.), Stern (H.), dir., Les ruses du salut. Religion et politique dans le monde indien, Paris, Editions de l'EHESS, 1995 et Kumar (R.), " The Historical Roots of Democratic India », in Kumar (R.), The Making of a Nation. Essays in Indian History and Politics, Delhi, Manohar, 1989.

5. J'emprunte le mot de “ chapelain " à L. Dumont qui précise : "Nous traduisons purohita par chapelain, mais il faut garder présente à l'esprit l'idée d'une délégation ou avant-garde spirituelle, presque d'un major ego » (Dumont (L.), « La conception de la royauté dans l'Inde ancienne ", in Dumont (L.), Homo hierarchicus, Paris, Gallimard, 1966, p. 353).

6. Dirks (N.), The Hollow Crown. Ethnohistory of an Indian Kingdom, Cambridge, Cambridge University Press, 1987, p. 4. 
européens ${ }^{7}$. Elle a disparu des nomenclatures officielles après l'indépendance de 1947 et l'instauration d'un régime politique sécularisé. Même si la constitution indienne ne fit explicitement référence au sécularisme qu'en 1976, à la suite du vote d'un amendement voulu par Indira Gandhi, telle fut bien sa philosophie dès sa promulgation en 1950. En Inde comme ailleurs, le rôle des conseillers spirituels a donc été mis en question après l'établissement de la République. Jawaharlal Nehru, Premier ministre de 1947 à 1964, a ostensiblement promu un style politique rationaliste et laïc. Il s'est toujours montré méfiant envers les religieux. Il a par exemple renforcé le rôle de l'Etat sur les temples hindous dont les revenus - issus de leurs biens fonciers et de dons parfois considérables - ont été directement gérés par l'administration pour éviter les détournements de fonds ${ }^{8}$. Par ailleurs, il a créé, en 1957, la Bharat Sadhu Samaj (1'Association des renonçants de l'Inde) qui avait vocation à regrouper les ermites et autres ascètes errants, dans lesquels le Premier ministre voyait volontiers des parasites, pour les utiliser dans la réalisation des plans quinquennaux ${ }^{9}$. La plupart des hommes politiques n'en ont pas moins continué à entretenir des liens étroits avec leurs guru, auprès desquels ils prenaient conseil et dont ils attendaient surtout une protection surnaturelle. C'était là une garantie supplémentaire pour la réussite de leurs entreprises. Cette relation était d'ordre privé mais elle pouvait être publicisée à l'occasion par un homme politique qui cherchait ainsi à acquérir un surcroît de légitimité, comme en témoigne la démarche d'Indira Gandhi.

\section{Les conseillers occultes d'Indira Gandhi en pleine lumière}

En dépit de son agnosticisme affiché - qu'elle héritait largement de son père - Indira Gandhi eut sans doute recours à des guru plus que tout autre homme (ou femme) d'Etat indien. Elle s'adressa tout d'abord à Ma Ananda Moyi, une femme bengalie dont les disciples avaient construit un ashram (sorte d'ermitage) à Dacca en 1931, mais qui avait passé sa vie à parcourir les routes de l'Inde jusqu'à sa mort en 1982. Elle consulta aussi Jiddhu Krishnamurti, un théosophe installé aux Etats-Unis mais qui retournait chaque automne en Inde. Elle sollicita surtout son aide au cours de l'état d'urgence, cette période dix-huit mois, entre 1975 et 1977, au cours de laquelle elle mit l'Etat de droit entre parenthèses pour résister aux poursuites judiciaires et à la montée des oppositions qui se manifestaient par

7. Hocart (A. M.), Rois et courtisans, op. cit.

8. Cf. le Report on the Hindu Religious Endowment Commission, 1960-1962, New Delhi, Ministry of Law [sans date]. Pour plus de détails, cf. Jaffrelot (C.), Tarabout (G.), "Les transformations de I'hindouisme ", in Jaffrelot (C.), dir., L'Inde contemporaine. De 1950 à nos jours, Paris, Fayard, 1997.

9. Hitavada, 15 avril 1956 et Statesman (Delhi), 27 mars 1957. 
de nombreuses manifestations de rue. Cette dérive autoritaire de sa pratique politique s'avéra très vite difficile à assumer pour Indira Gandhi. En proie au doute, elle chercha secours auprès de Krishnamurti. Nous savons, grâce aux confidences qu'elle a faites à Pupul Jayakar, une de ses amies intimes et une disciple de Krishnamurti, qu'elle dit un jour de 1976 à ce sage : « Je suis assise sur le dos d'un tigre [...]. Que dois-je faire? » Pupul Jayakar indique que Krishnamurti « refusa de la conseiller mais suggéra qu'elle prenne une vue d'ensemble de sa propre vie, de tous les conflits, des peines, des attaques qu'elle subissait, de ses actions, de ses erreurs et qu'elle considère toutes ces choses comme un seul problème pour agir ensuite avec détachement et sans crainte des conséquences ${ }^{10}$. "

La relation qu'Indira Gandhi entretenait avec Krishnamurti était d'abord d'ordre personnel, puisque la première cherchait auprès du second un réconfort psychologique et moral. Cette relation ne s'en inscrivait pas moins dans la logique du schéma traditionnel associant un dirigeant politique et un conseiller spirituel. La façon dont Indira Gandhi s'appliquait à rendre publique cette relation, qui relevait a priori du domaine privé, en apporte une confirmation : il s'agissait bien de se montrer avec un homme jouissant d'un grand prestige spirituel.

Dans le même temps, Indira Gandhi chercha à obtenir la caution d'un autre de ses guru, Vinoba Bhave, pour donner à l'état d'urgence un vernis de respectabilité. Bhave était alors l'un des derniers disciples de Gandhi encore en vie. Il tirait son prestige de son action passée auprès du Mahatma - celuici l'avait désigné dès 1921 à la tête de son ashram de Wardha, au Maharashtra - mais aussi de ses initiatives personnelles. Deux mois après la mort de Gandhi, en 1948, Bhave avait fondé la Sarvodaya Samaj (littéralement la «Société du service universel») à l'origine du mouvement bhoodan (littéralement "don des terres") au cours duquel il demanda aux propriétaires fonciers de céder une partie de leurs terres à des paysans pauvres. Dans ce but, il arpenta l'Inde à pieds pendant quinze ans avant de se retirer dans son ashram de Paunar où il séjournait avec ses disciples. Bien que reclus, Bhave était demeuré très présent sur la scène publique nationale, y jouant le rôle - selon l'expression consacrée des journalistes - de Sarkari guru (guru du pouvoir ${ }^{11}$ ). Indira Gandhi le consulta surtout dans les années 1970. Elle eut une longue réunion avec lui dans son ashram le 2 janvier 1974 et lui rendit à nouveau visite le 7 septembre 1975, quelques mois après qu'elle eut déclaré l'état d'urgence. Bhave avait déjà donné son approbation au nouveau régime en y voyant une "ère de discipline ». Il est apparu alors comme « le rishi [littéralement le voyant, l'homme pour lequel l'avenir n'a

10. Jayakar (P.), Indira Gandhi. A Biography, Delhi, Viking, 1992, p. 312.

11. Pour plus de détails sur Vinoba Bhave, cf. Bhave (V.), Mov'ed by Love. The Memoirs of Vinoba Bhave, Hyderabad, Sat Sahitja Sahayogi Sangh, 1994. 
pas de secret] qui a légitimé l'action de Madame Gandhi ${ }^{12}$ ». L'importance accordée au fait qu'il avait "donné sa bénédiction " au gouvernement - la presse, soumise à la censure, consacra de nombreux éditoriaux à cet événement - traduit bien la puissance du lien qui demeure en Inde entre le pouvoir temporel et l'autorité spirituelle.

Indira Gandhi ne manquait pas de se faire photographier avec ses différents guru, ce qui reflète son souci constant de donner le plus de publicité possible à ces rencontres. Si de tels rapports semblent à première vue relever de la "face cachée" du pouvoir, les hommes politiques y voient en effet une ressource. Ils ne sont pas seulement considérés comme légitimes; ils sont nécessaires à l'affirmation d'une légitimité politique. La forme ritualisée de ces rencontres mérite ici d'être soulignée. Elles doivent de préférence avoir lieu dans l'ashram du guru où les hommes politiques font donc l'effort de se déplacer. Ceux-ci commencent par baiser les pieds du maître, selon la formule de salut consacrée d'un supérieur par un inférieur, qui reçoit en échange sa bénédiction d'un geste de la main sur la tête. La suite, à la limite, importe peu : l'essentiel est d'avoir cette bénédiction, le darshan (la vision) du guru, et de pouvoir s'afficher à ses côtés. Un autre guru d'Indira Gandhi, Devraha Baba, se contentait d'ailleurs de lui donner cette bénédiction avec ses pieds. Indira Gandhi et son fils Rajiv ont ainsi été photographiés la tête couverte des pieds de cet homme frêle, assis devant sa cabane sur pilotis, au bord d'un fleuve sacré, affluent du Gange, la Yamuna. Leurs échanges s'arrêtaient là, mais la symbolique de ce geste était très forte et les photographies qui le mettaient en scène ont beaucoup circulé.

Malgré le caractère officiellement sécularisé de la République indienne, les "hommes de Dieu " n'ont donc pas disparu de l'entourage des hommes politiques qui, à l'instar d'Indira Gandhi - le cas entre tous le plus exemplaire - y puisaient un soutien psychologique et une ressource symbolique. D'où l'usage public qu'Indira Gandhi a fait de sa relation privilégiée avec plusieurs guru. Ce dévoilement d'une des faces cachées du politique n'est toutefois pas sans limite. Certains guru sont moins légitimes que d'autres. C'est notamment le cas des tantriques, surtout lorsque leurs activités occultes ont une dimension criminelle.

\section{Tantrisme et criminalité : la face cachée du politique}

Le tantrisme emprunte quelques-uns de ses éléments aux pratiques chamaniques mais il s'emploie surtout à réorganiser les éléments de l'hindouisme à travers de véritables rituels d'inversion dont le but ultime

12. Ostergaard (G.), Non Violent Revolution in India, New Delhi, Gandhi Peace Foundation, 1985, p. 221. 
demeure le salut (ou moksha) ${ }^{13}$. En lieu et place de l'ascétisme, il glorifie le désir, notamment sous la forme d'expériences sexuelles, et au lieu de prêcher la non-violence, il admet le sacrifice animal. Il est d'ailleurs associé à la transgression des interdits et à des pratiques de magie noire. Les pouvoirs des tantriques sont de ce fait utilisés en politique pour disqualifier un rival au moyen du "mauvais œil", ce qui rappelle certains usages de la sorcellerie en Afrique sub-saharienne. Indira Gandhi confia ainsi à Pupul Jayakar avoir reçu des "rapports secrets sur des rituels tantriques et de magie noire qui auraient été accomplis pour [la] détruire ainsi que [sa] santé ${ }^{14} »$. En 1982, elle fit appel à Krishnamurti pour la délivrer de ces sortilèges - ce que, selon elle, il réalisa avec succès. Indira Gandhi croyait donc à l'efficacité des rituels tantriques et à l'antidote qu'un homme comme Krishnamurti pouvait lui apporter ${ }^{15}$. Lors du procès de son fils cadet, Sanjay, que la justice poursuivit après l'état d'urgence pour plusieurs affaires de corruption, elle avait déjà sollicité des experts en tantrisme pour le protéger. Le récit de Pupul Jayakar est très explicite à ce sujet : en 1979, Indira Gandhi décida de :

"Réaliser le laskshachandi path, un rituel au cours duquel on récite cent mille versets pour invoquer les pouvoir originels et l'énergie de Chandi, la déessemère qui englobe la totalité de l'univers. Ces rituels se déroulaient dans le temple de Kali de Jhansi [au nord de l'Inde]. Les yagna [les sacrifices offerts au feu], les oblations et la lecture des versets se firent en secret de 1979 à 1983. C'était une période où je la voyais souvent, une période où elle parlait librement de ses problèmes, mais jamais au cours de nos très nombreuses rencontres et conversations, elle n'a révélé participer à ces rituels de vie en relation avec l'énergie féminine essentielle. Pendant qu'elle recherchait protection et pouvoir pour son fils dans les sanctuaires de Chandi, par des rituels secrets du chamanisme et du tantrisme, elle était aussi en contact avec J. Krishnamurti. Cela soulève des questions auxquelles il est impossible de répondre. A quel niveau impénétrable de son inconscient pouvait-elle tenir ensemble ces énergies contradictoires et explosives? L'une participait de la voie du seer [le sage], du chemin de la négation du pouvoir, de la compassion, de la libération, que l'on peut comparer à la marche sur le fil d'une épée ; l'autre, la voie de Chandi, de l'énergie féminine originelle, brandie comme le glaive du pouvoir. Ces deux forces cosmiques pouvaient-elles tenir dans une seule conscience? Et pourtant, à l'extérieur, méticuleusement, en parole et en action, elle suivait le sentier séculier, sans jamais manquer une marche, refusant de mêler son rôle public de fille de Jawaharlal Nehru, d'héritière d'un tempérament scientifique et rationnel, ses responsabilités de Premier ministre avec ses propres nécessités intérieures ${ }^{16}$. "

13. «La structure, identique en ses éléments, est entièrement bouleversée par une utilisation différente " (Biardeau (M.), L'hindouisme, anthropologie d'une civilisation, Paris, Flammarion, 1981, p. 162).

14. Les faits rapportés ici sont repris de Jayakar (P.), Indira Gandhi..., op. cit., p. 440 et s.

15. Biardeau (M.), L'hindouisme..., op. cit., p. 162.

16. Ibid., p. 470-471. 
La démarche d'Indira Gandhi montre qu'il existe en Inde, et ailleurs sans doute, trois rapports possibles entre hommes de religion et hommes politiques : au niveau officiel, c'est l'absence de relation qui l'emporte, c'est le discours de Nehru l'agnostique; au niveau officieux, mais pas illégitime, Indira Gandhi faire appel aux guru les plus licites, comme Krishnamurti ou Bhave; enfin, au niveau secret, apparaît Indira Gandhi, la disciple du tantrisme dont on ne sait presque rien. Indira Gandhi ne dit pas un mot de sa participation aux rituels tantriques à Pupul Jayakar, qui était pourtant sa plus proche confidente. Il s'agit là d'une des véritables faces cachées de la politique. Le tantrisme a des affinités particulières avec le pouvoir, comme en témoigne la place centrale qu'y occupe la notion de shakti, le principe féminin, présenté par le tantrisme comme le "principe actif dans l'univers ${ }^{17}$ ", que l'on traduit généralement par "énergie » ou " pouvoir », et qui se manifeste principalement par le culte de la déesse Kali ou Durga. La shakti, c'est le pouvoir. Indira Gandhi semble avoir été fascinée par cette équation. Elle était électrisée par les hymnes à la gloire de la déesse : le culte des shakta, le sapta sati, était pour elle "comme le tonnerre; il emplit les oreilles et le cœur, il pénètre tout ${ }^{18}$ ». Elle se percevait peut-être comme la shakti de l'Inde. En tout cas, elle a été assimilée à la shakti à plusieurs reprises dans l'imaginaire collectif indien. Atal Bihari Vajpayee, actuellement Premier ministre, l'a ainsi comparée à Durga après qu'elle a déclaré la guerre au Pakistan en 1971 pour conduire l'Inde à la victoire. Les affinités entre tantrisme et pouvoir (et plus précisément les pouvoirs des guru tantriques) expliquent que de nombreux politiciens aient recours à leurs services.

Indira Gandhi elle-même a fini par s'en remettre à l'un de ces guru, Dhirendra Brahmachari qui avait acquis dans les années 1950 la réputation d'un expert en yoga et s'était introduit par ce biais dans la haute société de Delhi. Il commença à enseigner le yoga à Indira Gandhi en 1958 et devint bientôt l'un de ses proches, ce qui lui valu d'être surnommé "Raspoutine ". Il se tenait très souvent à ses côtés, à tel point que même ses collaborateurs se plaignaient parfois de ne pouvoir lui parler seul à seul. Brahmachari affirma plus encore son rôle de chapelain du roi en officiant dans les rituels familiaux, comme les funérailles de Sanjay Gandhi, le plus jeune fils d'Indira, mort en $1980^{19}$. Il s'agissait pourtant d'un yogi d'un genre nouveau, un de ces guru modernes qui avaient embrassé la carrière religieuse à des fins fort peu désintéressées et qui s'efforçait d'ailleurs de gagner des fidèles dans l'élite sociale, voire à l'étranger. Un biographe d'Indira Gandhi indique ainsi que Brahmachari « conduisait une Toyota et pilotait son propre avion

17. Ibid., p. 162.

18. Cité dans Jayakar (P.), Indira Gandhi..., op. cit., p. 442.

19. Malhotra (I.), Indira Gandhi. A Personal and Political Biography, Londres, Hodder \& Stonghton, 1989, p. 188. 
privé qui lui avait été offert - il insistait particulièrement sur ce fait - par ses disciples étrangers ${ }^{20}$ ». Après la défaite électorale d'Indira Gandhi en 1977, Dhirendra Brahmachari fut la cible de bien des enquêtes judiciaires. Il fut notamment accusé d'avoir créé une fabrique d'armes illégale ${ }^{21}$. Mais les poursuites furent suspendues après le retour d'Indira Gandhi au pouvoir en 1980. Pour elle, la mauvaise réputation de Brahmachari, dont elle ne pouvait pas ne pas être avertie, n'était pas une raison suffisante pour rompre avec lui. Lorsque des proches insistaient pour comprendre ce qui l'amenait à garder un tel personnage dans son entourage, elle répliquait: «Il doit bien y avoir une raison! " Cette raison, pour Pupul Jayakar, ce ne pouvait être que l'obédience tantrique de Brahmachari : "Adepte de cette science ésotérique, il faisait partie de ces gens qui effrayaient Indira en évoquant de sombres rites tantriques accomplis dans des sanctuaires secrets par ceux de ses adversaires qui voulaient la détruire ainsi que Sanjay. Brahmachari lui parlait sans doute d'autres rites tout aussi puissants et de mantras [formules rituelles] qui pouvaient la protéger de ces forces maléfiques ${ }^{22}$. "Dhirendra Brahmachari ne sera finalement mis à la porte de la maison des Gandhi qu'après la mort d'Indira, en 1984, par Rajiv, son fils aîné. Mais d'autres tantriques continueront à s'insinuer dans les coulisses du pouvoir.

L'attrait qu'un autre de ces guru tantriques, Chandraswami, a exercé sur les hommes politiques de toutes obédiences au cours des vingt dernières années revêt ici une valeur exemplaire. Chandraswami avait commencé sa carrière politique comme leader des Jeunesses du parti du Congrès dans la région d'Hyderabad. Mais sa réputation de guru tantrique alla croissante dans les années 1970 et il abandonna la carrière politique pour devenir un conseiller plus ou moins " occulte " d'hommes politiques. Il justifiait cette association en se référant aux liens classiques entre les hommes de pouvoir et les hommes de Dieu dans l'hindouisme classique: "La tradition de ce pays veut que la royauté et la religion soient inextricablement mêlées. La relation entre le rajah [roi] et le rishi [voyant] et la relation entre la rajniti [politique] et le dharma sont comme la relation entre le corps et l'âme. Ils sont inséparables ${ }^{23}$. " Pourtant, en bon tantrique, Chandraswami était plus qu'un simple conseiller du prince. Il admettait détenir différents types de pouvoirs correspondant à trois types de rituels: le maha mrityunjay yajna (un rituel

20. Ibid.

21. Jayakar (P.), Indira Gandhi, Paris, Plon, 1994, p. 347.

22 . Ibid., p. 348.

23. Interview de Chandraswami, India Today, 31 octobre 1995. Devant la Commission Jain, chargée de l'enquête sur l'assassinat de Rajiv Gandhi, Chandraswami insistait systématiquement sur l'aspect spirituel de sa personnalité. Il déclarait que, en tant que sadhu (renonçant-ascète), il était de son devoir d'effacer de sa mémoire tout ce qui regardait la période antérieure à son retrait du monde. Il faisant remarquer que ses relations avec les hommes politiques étaient seulement guidées par des "raisons spirituelles" (Frontline, 3 novembre 1995). 
ayant pour but de prévenir la mort) ; le bagla mughi yajna (un rituel visant à éliminer ses ennemis); et le rudra mahayagya (un rituel destiné à porter préjudice à ses adversaires). Il a soutenu qu'il n'avait jamais utilisé ces deux derniers pouvoirs. Mais des journalistes qui ont enquêté dans son ashram ont rapporté que onze brahmanes s'y étaient rendus en son absence pour se livrer à un rituel particulier permettant de « nuire à ses ennemis de plusieurs manières sans entraîner aucune violence physique. Il s'agit simplement d'une prouesse mentale d'un tantrique, qui y aboutit grâce à une longue période de méditation [sadhana] ${ }^{24}$. " Les mantras qui étaient récités par les onze brahmanes servaient à ébahir les ennemis et à les étourdir (stambhan), à - perturber leur esprit (uchachadan) et à provoquer des conflits entre eux (idveshwan). Selon les disciples de Chandraswami, ce guru s'est adonné à cette pratique en de nombreuses occasions.

Que de tels épisodes soient vrai ou faux n'a que peu d'intérêt. L'important, c'est que Chandraswami ait cherché à maquiller derrière la tradition hindoue associant un roi à son chapelain, des pratiques du tantrisme et qu'en dépit de leurs dénégations publiques, bien des hommes politiques ont cherché à bénéficier de ses pouvoirs. Cette contradiction entre des attitudes privées et des discours public fut particulièrement évidente lors des auditions organisées par la Commission d'enquête présidée par le juge Jain, chargée de faire la lumière sur l'assassinat, en 1991, de Rajiv Gandhi. Chandraswami ayant été accusé par la police d'avoir joué un rôle dans cette affaire, le juge Jain interrogea les hommes politiques qui l'avaient fréquenté. En réponse à ses questions, un ancien Premier ministre, Chandra Shekhar, déclara : "J'ai été introduit auprès de lui [Chandraswami] en 1971 à New Delhi, par un ami ministre du parti du Congrès [dont il ne mentionne pas le nom], qui me l'a décrit comme une personne formidable qui avait des pouvoirs supranaturels. " Naturellement, Chandra Shekhar insiste sur le fait qu'il n'a jamais cru à ces allégations stupides : "Souvent, je lui ai demandé : "Quels sont tes pouvoirs tantriques?" Je voulais voir ces pouvoirs. J'ai rencontré de nombreux tantriques mais aucun ne $\mathrm{m}^{\prime} \mathrm{a}$ jamais montré ses pouvoirs ${ }^{25}$. " Pourtant, Chandra Shekhar a cherché à être associé avec Chandraswami, comme un grand nombre d'hommes politiques ${ }^{26}$. Un autre

24. Les événements dont il est question ici, ainsi que les citations, sont repris de The Pioneer, 15 octobre 1995.

25. The Times of India, 18 avril 1996.

26. L'ampleur du réseau des disciples de Chandraswami se manifesta de manière éclatante en 1996, lors des noces de la fille de Vidya Charan Shukla, qui avait été ministre d'Indira Gandhi, de Rajiv Gandhi et de Narasimha Rao. Les reportages dans la presse mentionnent le grand nombre de personnes - hommes politiques inclus - qui " faisaient la queue pour toucher ses pieds » en signe de respect et pour recevoir sa bénédiction (The Times of India, 24 avril 1996). Les funérailles de la mère de Chandraswami furent aussi l'occasion pour nombre d'hommes politiques indiens, certains ayant - ou ayant eu - de très hautes fonctions, de rencontrer le guru (Statesman (Delhi), 21 avril 1993). Ceci révèle un autre aspect des rapports entre le privé et le 
ancien Premier ministre, Narasimha Rao, fut plus proche encore de lui. Chandraswami a déclaré devant la commission Jain que, lorsqu'il allait le voir à sa résidence officielle, sa voiture n'était pas contrôlée, ce qui était un privilège très exceptionnel. Lors de la même audition, il a cependant protesté contre les allégations selon lesquelles il avait fait usage de yagya [rituels sacrificiels] pour permettre à Rao de devenir Premier ministre 27 .

Le contraste entre Chandraswami d'un côté et Vinoba Bhave ou Krishnamurti de l'autre, est patent. Chandraswami prétend s'inscrire comme eux dans le schéma hindou traditionnel en y remplissant des fonctions de conseiller spirituel des hommes de pouvoir mais ces derniers lui dénient ce rôle en public. S'ils recourent à ses services en secret, il ne peuvent se prévaloir de cette relation. Chandraswami est frappé d'opprobre en tant que tantrique, en tant qu'adepte de pratiques à la réputation sulfureuse. C'est pourquoi la commission Jain rencontra un écho considérable dans la presse: elle dévoilait des attitudes illicites et délégitimait du coup une partie de la classe politique. Aucun personnage public n'avait intérêt à révéler cet aspect-là de la face cachée du politique et tous ont nié d'ailleurs avoir cru en ses pouvoirs. Naturellement, Chandraswami était d'autant plus violemment rejeté par les hommes publics qu'il était soupçonné d'avoir participé au complot contre Rajiv Gandhi et que ses activités comportaient une dimension criminelle.

Chandraswami se situe en effet dans la part d'ombre du politique non seulement en tant que tantrique, mais aussi en tant que " mafieux » ayant su jouer de son statut de conseiller spirituel pour servir d'intermédiaire dans des affaires douteuses. Ayant des disciples dans les milieux d'affaires - et dans ceux de la contrebande internationale - autant qu'au sein de la classe politique indienne, il était bien placé pour s'entremettre dans les transactions de ce monde interlope. Il a ainsi organisé des opérations financières pour le compte de son disciple Adnan Khashoggi, un marchand d'armes saoudien, et, par l'intermédiaire de ce dernier, pour son ami le sultan de Brunei ainsi que pour les frères $\mathrm{Al}$ Fayed, des hommes d'affaires égyptiens (il est notamment intervenu dans l'achat par ces derniers du magasin Harrods à Londres). Il a recueilli de l'argent - parfois de l'argent sale - provenant de riches Indiens de la diaspora (y compris le magnat du " pickles » Lakhubhai Pathak), qui ont eu recours à ses services pour approcher des hommes politiques faisant partie de ses disciples dont il leur fallait l'accord - au besoin en échange de pots de vin transitant par Chandraswami - pour opérer, voire investir, en Inde. Enfin et surtout Chandraswami a été accusé d'avoir participé à des opérations secrètes dirigées contre Rajiv Gandhi comme il a déjà été noté plus haut : il a été désigné comme étant l'un

public en Inde : les cérémonies privées, comme les mariages ou les enterrements, sont l'occasion pour les hommes politiques de se montrer et de rencontrer leurs rivaux.

27. The Times of India, 11 mars 1996. 
des proches associés du président Giani Zail Singh qui avait cherché à démettre Rajiv Gandhi de son poste de Premier ministre; plus important encore, il a été soupçonné d'avoir joué un rôle dans l'assassinat de celui-ci, sans toutefois que la Commission Jain n'ai jamais pu en faire la preuve. Chandraswami, à l'instar de Dhirendra Brahmachari n'était n'est donc pas seulement appelé à rester un homme de l'ombre en raison de ses seules pratiques tantriques mais aussi du fait de ses liens avec le monde du crime. Une telle combinaison n'est pas rare et ne concerne d'ailleurs pas seulement les tantriques. Ceux-ci sont sans doute les plus susceptibles de nager dans les eaux troubles de la criminalité mais la tentation peut être grande pour d'autres guru de procéder de la même manière. Leur accès privilégié aux puissants attire en effet tous ceux qui voudraient user de leur médiation pour obtenir des faveurs indues. Ils peuvent d'ailleurs jouer de leur influence de leur propre initiative, au profit d'un tiers ou d'eux-mêmes, tout en feignant le plus grand désintéressement.

Contrairement à l'hypothèse d'Ashis Nandy, l'idéal d'austérité n'est sans doute pas le principal répertoire politique de l'Inde contemporaine. Il a pu jouer un rôle prépondérant à l'époque coloniale en raison de l'action de Gandhi. Mais dans les situations courantes, la sphère spirituelle est séparée de celle du pouvoir politique, la première s'incarnant dans la figure du Brahmane (le guru) et la seconde dans celle du roi (kshatriya). Nehru a proscrit cet arrangement politique dans le cadre de la sécularisation de l'Etat, mais il est réapparu au plus haut niveau avec Indira Gandhi. S'il ne s'agissait pas là d'un phénomène officiel, des hommes politiques ont pu juger opportun de rendre ce secret public pour bien montrer qu'ils étaient en étroite relation avec des sages, conformément à la configuration traditionnelle du pouvoir. Les seuls guru à ne jouir d'aucune légitimité naturelle et, par conséquent, à rester dans l'ombre, sont les tantriques qui, non contents d'être versés dans la magie noire, entretiennent des relations inavouables avec l'univers des trafiquants et des criminels. Dans ce cas, la révélation de secrets comme ceux auxquels s'est attaquée la Commission Jain avec Chandraswami, a des effets de délégitimation radicale dont Narasimha Rao fut une des principales victimes. Au-delà de la relation interindividuelle entre un homme de pouvoir et son guru, la question des conseillers occultes du pouvoir et de leur mise à jour se pose aujourd'hui en Inde à propos de la mouvance nationaliste hindoue, au sein de laquelle la notion de guru a été réinventée et entretient des relations là aussi ambiguës avec la sphère publique.

\section{Le RSS, «Raj guru »'un genre nouveau}

Dans l'hindouisme, le guru est d'abord un principe, qui s'incarne généralement dans un homme ou une femme, mais qui peut aussi prendre 
une forme très différente. L'épopée du Mahabharata relate ainsi comment un jeune "hors caste", Eklavya, auquel aucun guru ne voulait enseigner l'art du tir à l'arc - réservé aux seules castes guerrières - modela l'effigie d'un guru et s'entraîna avec grand profit sous son « regard ", signe que l'efficacité de la relation avec le maître relève d'abord de la subjectivité du disciple. Cette malléabilité de la notion de guru a facilité sa sécularisation, comme en témoigne le rôle que joue depuis cinquante ans le RSS (Rashtriya swayamsevak sangh, Association des volontaires nationaux) dans les coulisses du pouvoir.

Le RSS a été créé en 1925 par des hindous de hautes castes - en majorité des brahmanes - qui s'inquiétaient des tendances panislamiques des musulmans de l'Inde et de l'impact culturel de la colonisation britannique - notamment en termes de conversion au christianisme ${ }^{28}$. Pour mieux défendre l'hindouisme, son fondateur, Keshav Baliram Hedgewar, a choisi de le moderniser en s'inspirant des points forts de ses ennemis, mais avec le souci constant d'inscrire ces importations dans un cadre familier à travers une véritable "vernacularisation » des emprunts en question. C'est ainsi qu'il a doté le mouvement d'un uniforme imité de la police anglaise, mais que cette allure paramilitaire est allée de pair avec une discipline découlant des arts martiaux de l'hindouisme. Hedgewar voulait développer un sens de la nation hindoue en s'inspirant des symboles patriotiques en usage à l'Ouest comme le drapeau que les militaires y saluent en signe de respect et d'allégeance nationale. Il ne se contenta pas de doter le mouvement du drapeau safran (la couleur de l'hindouisme) de Shivaji, un chef de guerre du Maharashtra qui avait lutté contres les Moghols au XVIII siècle, mais fit de ce Bhagva Dhwaj (littéralement le "drapeau safran") le guru du mouvement ${ }^{29}$. Pour lui, c'était une façon d'indiquer qu'il ne serait pas le guru du RSS, que la source d'inspiration devait venir de ce drapeau, symbole de la nation dont le RSS était l'avant-garde. Tous les matins et tous les soirs, les membres des branches (shakha) locales du RSS s'assemblent à travers l'Inde pour des séances d'entraînement physique et de propagande idéologique sous l'œil de ce guru imaginaire. Chaque session commence en effet par l'installation d'un drapeau que tous saluent et qui "veille » ensuite sur le bon déroulement des exercices, tout comme Eklavya s'entraînait sous le « regard » d'un guru inanimé.

Dans le cas du RSS, la métaphore du guru dépasse cependant le cas du drapeau car le RSS se définit lui-même comme un guru collectif, non seulement parce qu'il est littéralement le porte-drapeau de la nation, mais parce qu'il peut se prévaloir de qualités spirituelles d'exception. Hedgewar a

28. Pour plus de détails, cf. Jaffrelot (C.), The Hindu Nationalist Movement and Indian Politics, New Delhi, Penguin, 1999.

29. Andersen (W.), Damle (S.), The Brotherhood in Saffron. The Rashtriya Swayamserak Sangh and Hindu Revivalism, New Delhi, Vistaar Publications, 1987, p. 90. 
d'emblée fixé aux cadres du mouvement - qui sont notamment chargés des shakha - une discipline de vie qui reproduisait des traits essentiels du renoncement (ou sanyas): non seulement ils devaient s'abstenir de toute activité professionnelle et ne pas fonder de foyer, mais il leur fallait aussi renoncer à s'installer en un lieu fixe, leur dévouement au mouvement impliquant une vie itinérante. Ils devaient en outre s'astreindre à une véritable ascèse, leur logement étant nécessairement spartiate et leur régime alimentaire des plus frugal. Hedgewar voulait faire de ces prêcheurs (pracharak) de véritables karma yogi (l'idéal du karma yogi est celui de l'homme qui s'adonne au yoga de l'action). Une telle discipline consiste à mener son activité mondaine dans le plus grand détachement : les fruits de l'action pour soi importent peu.

Le RSS s'est étendu à mesure que s'étoffait le corps des pracharak et que leur réseau de shakha se densifiait. Dans le même temps le mouvement a revêtu des allures sectaires aux deux sens du terme. D'une part, il a présenté certains traits des sectes hindoues puisqu'au-delà de la discipline austère de ses cadres, il s'est efforcé d'abolir les distinctions de castes en son sein - les sectes de l'hindouisme répondent à une vocation égalitaire - et s'est doté d'un rituel complexe incorporant son propre calendrier de fêtes et autres célébrations commémoratives. D'autre part, son nationalisme hindou a pris une tournure xénophobe qui s'est traduite par le rejet violent des minorités musulmane et chrétienne. Le RSS a donc très vite éveillé les soupçons des Britanniques à l'époque coloniale, non seulement en raison de sa dimension paramilitaire mais aussi des violences auxquelles il s'adonnait volontiers contre les musulmans et les chrétiens. Le RSS en a d'autant plus cultivé le goût du secret. Il a encore perfectionné ses techniques de fonctionnement clandestin au lendemain de l'indépendance de 1947 après que l'un de ses anciens membres, Nathuram Godse, eut assassiné le Mahatma Gandhi qu'il tenait responsable de la partition de l'Inde et de son cortège d'émeutes intercommunautaires. Nehru a alors interdit le mouvement pendant deux ans en considérant qu'il n'avait pas droit de cité dans un régime séculariste, sauf à réformer ses pratiques. Le RSS s'est doté de statuts compatibles avec le régime mais s'est vu contraint à la discrétion, voire à la clandestinité. Indira Gandhi a en effet interdit à nouveau le RSS en 1975 au moment de l'état d'urgence au motif qu'il s'agissait d'un mouvement antiséculariste ("communaliste», pour reprendre l'expression consacrée en Inde). Nombre de ses cadres entrèrent alors dans la résistance clandestine. Ces interdictions récurrentes ont donc conforté le RSS dans un mode de fonctionnement rompu aux techniques $d u$ secret. Mais sa vocation première le plaçait de toute façon en retrait de la sphère publique, dans son ombre ou, plus précisément, dans ses coulisses, d'où il pouvait animer la scène.

Le successeur d'Hedgewar, Madhav Shiv Golwalkar, présentait en effet le RSS comme un mouvement social destiné à influencer le politique, à être sa 
source d'inspiration religieuse. Reprenant à son compte l'agencement traditionnel du politique en Inde, il soulignait volontiers que « les dirigeants politiques n'ont jamais fourni ses critères à la société. Ils n'ont jamais été les piliers de notre vie nationale. Les saints et les sages, qui se sont élevés audessus des tentations mondaines de la richesse et du pouvoir et qui se sont consacrés à l'établissement d'une société heureuse, vertueuse et intégrée, ont toujours été ses hérauts. Ils représentaient la dharamsalta [l'autorité du dharma]. Le roi ne faisait que se conformer avec ardeur à cette autorité supérieure $^{30}$. » Or, précisément, le RSS se veut formé de renonçants dans le monde, de karma yogi qui ont surmonté les tentations au moyen d'une discipline ascétique. C'est pourquoi Golwalkar et les hommes qui lui ont succédé à la tête du RSS ont prétendu constituer ce dernier en un véritable Raj guru. L'un des idéologues du mouvement les plus influents, K.R. Malkani, définit ce rôle comme celui d'un "conseiller moral"31". Golwalkar s'inscrivait bien dans une telle perspective lorsqu'il déclarait: «Nous aspirons à devenir ce creuset radieux de tous les idéaux que notre société chérit depuis la nuit des temps - tout le pouvoir indescriptible qui rayonne à partir du soleil. Alors le pouvoir politique, qui tire sa force de cette source de la société, n'aura d'autre [objet] que de refléter ce même rayonnement ${ }^{32}$. "

Le RSS a d'abord cherché à se poser en conseiller du prince auprès des gouvernants du Congrès, mais ceux-ci ne partageaient pas ses vues. Aussi s'est-il résigné à participer à la création d'un parti politique, le Bharatiya jana sangh (Association du peuple indien) à l'occasion des premières élections générales, en 1952. Cette formation a rapidement été prise en main par des pracharak du RSS qui restaient très liés à la "maison mère ». Le BJS a plafonné à $10 \%$ des voix jusqu'aux années 1970. En 1977, il a fusionné avec d'autres partis au sein du Janata party, une formation qui, profitant de l'impopularité de l'état d'urgence, renversa aussitôt le Congrès d'Indira Gandhi. Les députés issus du BJS étaient en grand nombre au Parlement et trois d'entre eux - dont Atal Bihari Vajpayee, l'actuel Premier ministre et Lal Kishna Advani, l'actuel ministre de l'Intérieur - entrèrent au gouvernement. Le RSS pouvait enfin espérer jouer les Raj guru. L'organe de presse du RSS en anglais, Organiser, se proposa ainsi d'aider le gouvernement à "élever la conscience nationale et sociale du peuple ${ }^{33} »$. Surtout, le successeur de Golwalkar, Babasaheb Deoras, s'employa à rencontrer les ministres importants - et pas seulement ceux de l'ancien BJS - pour les gagner à sa cause. Les nationalistes hindous soutinrent à ce moment-là trois dossiers bien précis. Ils voulaient une loi nationale abolissant l'abattage des vaches

30. Golwalkar (M. S.), Bunch of Thoughts, Bangalore, Jgaramna Prakashan, 1966, p. 93.

31. Entretien avec l'auteur de K. R. Malkani, 16 novembre 1989, New Delhi.

32. M. G. Golwalkar, Bunch of Thoughts, op. cit., p. 103.

33. Organiser, 28 mai 1977. 
(la vache étant un animal sacré de l'hindouisme que les adeptes d'autres religions consomment parfois). Ils souhaitèrent aussi mettre au point une législation réglementant très rigoureusement les conversions afin de limiter l'action des missionnaires. Enfin, ils demandèrent la mise à l'index de certains manuels d'histoire utilisés dans le système éducatif et dont les auteurs étaient accusés de "distorsions marxistes", de sous-évaluer le rôle passé du RSS, de ne pas souligner assez les exactions ayant accompagné les invasions musulmanes et de souscrire, à tort, à la thèse de l'invasion aryenne (car les aryens ne peuvent être, pour les nationalistes hindous, que les autochtones de l'Inde).

Les pressions exercées par le RSS dans son soi-disant rôle de Raj guru indisposèrent rapidement les membres du Janata party qui n'étaient pas issus du BJS mais de la mouvance socialiste ou même de scissions du Congrès. Le RSS fut accusé de convoiter un statut inacceptable en démocratie, celui d'un parti extra-constitutionnel : comment un groupe ne s'étant pas soumis au verdict des urnes pouvait-il chercher à influencer ainsi la sphère publique à travers ses représentants au sein du système politique? La question ne se serait pas posée si ce groupe n'avait entretenu des relations privilégiées avec une partie des parlementaires et certains ministres. Ceux-ci furent accusés de «dual membership » ou double allégeance : ils ne pouvaient à la fois être les élus d'un parti, le Janata, qui avait son propre programme, et les membres d'un mouvement dont l'idéologie était bien différente. Ce conflit provoqua l'éclatement du Janata party et la chute du gouvernement en 1980. Les leaders du RSS en conçurent une immense amertume, d'autant plus que les anciens du BJS reconstituèrent un parti, le Bharatiya janata party (Parti du peuple indien) qui, pour commencer, prit ses distances avec lui.

Cette attitude était purement tactique car les chefs du BJP continuaient d'apparenter le RSS à un guru collectif. En 1994, le président du parti, Lal Kishna Advani, déclarait ainsi : "Les sadhus et les renonçants, ou encore le RSS, exercent sur nous une influence morale et éthique. Après tout, il y a des milliers de pracharak qui sont nos pairs, nos égaux en tout, qui ne manifestent jamais la moindre ambition. C'est pourquoi nous les admirons ${ }^{34}$. 》 En 1994, ce genre de considération ne pouvait guère être formulée que dans le huis clos d'un entretien en tête à tête, car la hantise d'une nouvelle campagne dénonçant le lien entre le BJP et le RSS marquait encore les esprits de la mouvance nationaliste hindoue. Lorsqu'un leader du BJP était mis à la question publiquement, il rétorquait systématiquement que son parti poursuivait des objectifs politiques bien différents du RSS - qui était généralement présenté comme une organisation culturelle, voire une association caritative. Il soutenait coûte que coûte qu'à aucun moment, le RSS n'influençait la démarche politique du BJP. En pratique, si le RSS ne

34. Entretien avec l'auteur de L. K. Advani, 11 février 1994, New Delhi. 
dicte pas sa conduite au BJP de façon quotidienne, il en inspire cependant les grandes lignes. Les occasions d'une telle collaboration ont longtemps été tenues secrètes, ou du moins les réunions étaient-elles organisées dans la plus grande discrétion. Il en allait ainsi de la conférence annuelle au cours de laquelle le chef du RSS rencontrait les députés du BJP. Cette conférence avait lieu lors de la fête de Guru dakshina. Dans l'hindouisme, Guru dakshina désigne la cérémonie au cours de laquelle les disciples d'un guru honorent leur maître à l'occasion de son anniversaire au moyen d'un don (dakshina). Le RSS a réinterprété cette institution traditionnelle. Pour lui, la Guru dakshina est la fête au cours de laquelle les membres du mouvement rendent hommage au drapeau safran - le guru du RSS - et lui font un don (dakshina), sorte de cotisation servant à financer le mouvement. Le fait que le chef du RSS rencontre les députés du BJP le jour de Guru dakshina est hautement symbolique: cela tend à en faire le guru de ces figures politiques du nationalisme hindou, bref un Raj guru.

Si cette relation entre le BJP et le RSS est longtemps restée dans l'ombre, elle tend à être affirmée publiquement depuis l'accession au pouvoir de ce parti en 1998. Cette publicisation nouvelle de la relation entre le RSS et le BJP est d'ailleurs apparue de manière éclatante lors de la cérémonie d'investiture du second gouvernement Vajpayee en 1999. Lorsque le BJP avait été en mesure de former le gouvernement pour la première fois en 1998, le RSS s'était montré fidèle à sa légendaire discrétion. En 1999, par contre, deux de ses leaders, H.V. Seshadri (le secrétaire général du mouvement) et K.S. Sudarshan (qui devait prendre la tête de l'organisation en mars 2000) se trouvaient au premier rang des invités lors de la cérémonie d'investiture. $C^{\prime}$ est donc sous leurs yeux que le Premier ministre et les autres membres du gouvernement prêtèrent serment sur la Constitution, en réponse au maître de cérémonie, le président de la République. Le RSS apparaissait d'autant plus en acteur public du jeu politique que des ministres issus du mouvement vinrent présenter leurs respects à Seshadri et Sudarshan, certains en faisant pranam (l'acte de toucher les pieds puis sa propre poitrine) pour recevoir leur bénédiction. Aucun organe de presse ne s'est ému de cette allégeance qui, vingt ans plus tôt, avait suscité le débat que l'on sait à propos du «dual membership ». Ce changement d'attitude tient peut-être au fait que le RSS avait commencé lui-même à jouer le jeu de la publicité. En avril 1998 - un mois après la formation du premier gouvernement Vajpayee - la réunion du conseil représentatif du RSS, l'Akhil bharatiya pratinidhi sabha (Assemblée pan-indienne des représentants) avait, pour la première fois, été ouverte aux journalistes. Les leaders du RSS s'y étaient montrés chaleureux - il s'agissait visiblement d'une opération de relation publique - et sûrs d'eux. Sudarshan avait répondu à un journaliste incrédule qu' « avec un gouvernement qui ne nous est pas hostile, nous allons pouvoir travailler mieux 35 ». "Travailler

35. Cité dans Frontline, 24 av ril 1998, p. 117. 
mieux " signifiait, notamment, exercer plus ouvertement encore un rôle de conseiller du pouvoir. Dès juillet 1998, le chef du RSS, Rajendra Singh, fut ainsi en mesure de présider une réunion à laquelle avaient été conviés les responsables de l'administration de l'Uttar Pradesh, le plus grand Etat de l'Inde. L'assistance comprenait notamment le Chief Secretary (le chef de l'administration de l'état) et le directeur général de la police de l'Uttar Pradesh. Rajendra Singh ouvrit la séance par une véritable conférence sur le thème " nationalisme et honnêteté ", son sujet de prédilection, qu'il aborda sous l'angle attendu des vertus du nationalisme et de la nécessité d'être vertueux pour renforcer la nation ${ }^{36}$. Rajendra Singh était là dans son rôle de Raj guru: celui-ci n'a pas seulement vocation à conseiller le gouvernement mais aussi l'administration qui en est le bras armé.

Le RSS cherche aussi à exercer ce genre d'influence auprès de différents ministères dont celui de l'éducation afin de transformer le contenu des enseignements et notamment les manuels $d$ 'histoire. Le ministre de l'Education et de la Recherche, Murli Manohar Joshi, un ancien cadre du mouvement, se prête volontiers à ces manœuvres. Il a nommé un ancien pacharak, Krishna Gopal Rastogi, au National Council for Educational Research and Training ${ }^{37}$. Peu après, l'enseignement du marxisme a disparu du programme de science politique d'une des filières du secondaire, ce qui a suscité une telle vague de protestations au Parlement que le gouvernement a du faire marche arrière ${ }^{38}$.

Il n'en demeure pas moins, en dépit de cette reculade, que l'influence du RSS sur le pouvoir est de plus en plus grande sans susciter pour autant les mêmes protestations indignées que dans les années 1970 et 1980 . Cette évolution tient sans doute au fait que le mouvement se sent protégé par le pouvoir, comme le confiait Sudarshan lui-même. Mais aussi au fait que si le nationalisme hindou est au pouvoir, c'est que ses idées trouvent un écho croissant dans la société. Certes, seul un cinquième des électeurs ont voté pour le BJP en 1999 et, nuance supplémentaire, voter pour le BJP ne signifie pas nécessairement soutenir le RSS dont les positions sont plus radicales encore. Mais cette évolution du corps électoral reflète bien une adhésion croissante de l'opinion au programme nationaliste hindou. Du coup, il semble moins nécessaire au RSS de rester dans l'ombre. Mieux, il peut être utile au BJP d'afficher la relation qu'il entretient avec ce mouvement. La démarche du Premier ministre Vajpayee revêt à cet égard une valeur exemplaire. Atal Bhari Vajpayee, déjà à l'époque où il servait de bras droit

36. Hindustan Times, 27 juillet 1998.

37. Rastogi est l'auteur d'une autobiographie - préfacée par Sudarshan - qui a suscité bien des réactions, car il y racontait comment, au moment des émeutes de la partition entre hindous et musulmans, il avait abattu de sang-froid une femme musulmane pour la sauver des violences d'une foule hindoue en furie (The Asian Age, 25 octobre 1999).

38. Frontline, 26 novembre 1999. 
au fondateur du Jana sangh, Shyam Prasad Mookerjee, faisait figure de modéré au sein de la mouvance nationaliste hindoue. Année après année, il s'est construit une image d'homme de compromis qu'il a peaufinée encore lorsque, ministre des Affaires étrangères du Janata party, il s'est montré ouvert vis-à-vis du Pakistan, facilitant l'obtention de visas aux musulmans indiens ayant de la famille de l'autre côté de la frontière. Le BJP a joué de cette image, même lorsque Vajpayee a été remplacé en 1986 à la tête du parti par un homme au discours plus militant, L.K. Advani : le parti savait qu'il aurait besoin d'un leader capable de gagner des voix au centre. De fait, Vajpayee est devenu son candidat au poste de Premier ministre lorsque le BJP est arrivé en tête aux élections, mais loin de la majorité absolue, ce qui nécessitait l'appoint de partenaires au Parlement. Il revint à Vajpayee, le plus diplomate des nationalistes hindous, de constituer une coalition qui regroupe aujourd'hui dix-huit formations.

Soucieux de cultiver son image, Vajpayee a longtemps évité de se montrer en compagnie des membres du RSS. En 1979, lors du débat sur le «dual membership", il a même refusé de s'asseoir au premier rang lors d'un meeting du RSS qui était organisé pour protester contre le mauvais procès dont l'organisation se disait victime ${ }^{39}$. Il a changé de stratégie depuis qu'il est au pouvoir. On l'a vu se laisser photographier, lors de manifestations du RSS, en train de faire le salut que les swayamsevak adressent au drapeau dans les shakhas (main droite à hauteur de la poitrine, paume vers le bas). Nuance importante, il ne s'affiche jamais revêtu de l'uniforme du mouvement.

Une telle démarche s'explique naturellement par la popularité croissante du nationalisme hindou mais elle procède également d'autres considérations. Vajpayee ne participe pas systématiquement en public aux réunions du RSS. Lorsqu'il se livre à cet exercice, c'est qu'il cherche à faire passer un triple message. C'est d'abord un moyen de rassurer les cadres du RSS (et du BJP) qui se sont souvent inquiétés de la distance qu'il entretenait avec leur mouvement. Ensuite, s'afficher aux côtés de leaders du RSS permet d'indiquer aux électeurs du BJP que, si la logique des coalitions l'empêche de mettre en œuvre l'intégralité du programme de son parti, son ancrage idéologique demeure intact. C'est enfin un moyen de faire savoir aux uns et aux autres qu'il n'est pas dupe des fastes du pouvoir; qu'il reste un swayamsevak avec ce que cela implique de dévouement et de simplicité, donc d'intégrité. Valoriser sa relation avec le RSS est en effet pour Vajpayee une manière de dire "je ne suis pas corrompu » en jouant sur l'image dont jouit le RSS au-delà des seuls partisans du nationalisme hindou.

Les relations qu'entretiennent les hommes politiques avec leurs guru continuent d'occuper en Inde une place centrale dans le dispositif du pouvoir en dépit de la sécularisation du régime et même si l'identité des

39. India Today, 16 mars 1979, p. 27. 
acteurs a changé avec le temps. Le couple formé par l'homme de pouvoir et par l'homme de Dieu, hérité de l'agencement des sommets de l'Etat à l'époque de l'Inde classique, a perduré à la période contemporaine, comme en témoignent les rapport d'Indira Gandhi avec ses guru. Mais le Raj guru a aussi pris les traits de tantriques et surtout, l'apparence du RSS, sorte de conseiller collectif du pouvoir. Du coup, le rapport de cet ordonnancement du pouvoir à la sphère publique a lui aussi évolué. Alors que, nonobstant le caractère laïc de la constitution, des leaders politiques pouvaient se montrer en public avec leurs conseillers spirituels - et même en tirer un bénéfice en termes de popularité lorsque ceux-ci s'appelaient Vinoba Bhave ou Jiddhu Krishnamurti - une telle publicisation devenait contre-productive lorsque les intéressés s'adonnaient au tantrisme et plus encore lorsqu'ils étaient impliqués dans des affaires troubles. La levée du secret, dans ce cas, s'accompagnait d'une délégitimation assurée des hommes politiques concernés.

Les relations du RSS et du gouvernement BJP dessinent un cas de figure plus complexe, non seulement parce qu'elles concernent deux institutions et non plus deux individus, mais aussi parce que leur publicisation est à géométrie variable. Dans un premier temps, l'opprobre qui frappait le RSS - du fait de son discours xénophobe et de ses responsabilités dans l'assassinat du Mahatma Gandhi - a amené cette organisation à se réfugier dans le secret et "son » parti politique à garder ses distances. L'acceptabilité croissante des thèses nationalistes hindoues et l'image d'intégrité que le RSS s'est construite à force d'austérité et de travail social ont cependant peu à peu changé la donne. De sorte que le Premier ministre Vajpayee peut trouver utile de s'afficher avec des leaders du RSS qui prétendent être la conscience du pouvoir. Vajpayee a d'ailleurs déclaré au cours de la dernière campagne électorale, "le RSS est mon esprit (soul) ", écho saisissant à la configuration des sommets de l'Etat à l'époque classique, comme si les zélateurs de la tradition hindoue étaient effectivement en mesure de rétablir un ordre politique où le gouvernement serait assorti d'un Raj guru. 